\title{
EMERGENCY VALVE PRESERVING ASCENDING AORTA REPLACEMENT FOLLOWING IATROGENIC RIGHT CORONARY ARTERY DISSECTION: A LIFE-SAVING PROCEDURE
}

\author{
Vidur Bansal ${ }^{1}$, Anand Mishra ${ }^{2}$, RUPESH KUMAR², Bhupesh Kumar², Parag Barwad ${ }^{2}$, \\ Nirupam Chakraborty ${ }^{2}$, and RUCHIT PATEL ${ }^{2}$ \\ ${ }^{1}$ PGIMER \\ ${ }^{2}$ Post Graduate Institute of Medical Education and Research
}

May 6, 2020

\begin{abstract}
Iatrogenic aortocoronary dissection is a rare but potentially fatal complication of coronary catheterizations. Although the incidence is comparatively low, dissection often leads to procedure failure with increased risk of myocardial infarction and death. Iatrogenic aortocoronary dissection is principally caused by disruption of intima at the ostia of the right or left coronary artery during interventional procedures and appears as luminal filling defects, the persistence of contrast or intimal tear outside the coronary lumen. Dissection could propagate in the anterograde direction causing subtotal or total occlusion of the coronary lumen or extend in the retrograde direction into the sinus of Valsalva, ascending aorta, aortic arch or descending aorta resulting in hemodynamic instability. We present a case of Right Coronary Artery dissection leading to Type-A aortic dissection suffered during diagnostic coronary catheterization. This required emergency supracoronary replacement of the ascending aorta with an aortic interposition tube graft and venous grafts to coronary arteries
\end{abstract}

\section{INTRODUCTION}

Iatrogenic aortocoronary dissection(IACD) is a fatal complication occurring in $0.02-0.04 \%$ cases $^{1-2,6}$ of coronary catheterization that requires early recognition and emergent surgical treatment. Aortic dissection may lead to serious complications like myocardial infarction or sudden cardiac death. Most described cases occurred during catheterization and angioplasty of the Right Coronary Artery(RCA) ${ }^{3-4}$. The morphological and structural differences between right and left coronary arteries may explain the fact that left aortic sinus is less prone to iatrogenic dissection ${ }^{5}$. Various options have been tried by many in the past including Coronary Artery Bypass Grafting(CABG) with aortic dissection repair, stenting the coronary artery, monitoring the aortic dissection using Transesophageal echocardiography(TEE), implanting coronary stents to maintain coronary blood flow before surgery and repairing aortic dissection surgically. Our patient had RCA Dissection which was extending to the ascending aorta with intramural hematoma. Reverse saphenous vein graft(RSVG) was used to revascularize the RCA with supracoronary replacement of the ascending aorta. Because of the significant disease in the proximal Left Anterior Descending Artery(LAD), a separate RSVG-LAD graft was also done.

\section{CASE SUMMARY}

A 63-year-old female, a known case of hypertension presented to the Emergency Department with the diagnosis of RCA dissection extending to the ascending aorta following diagnostic coronary catheterization 
at a local center done for evaluation of angina. As per records coronary catheterization was performed via the right trans-radial approach. Coronary catheterization(Figure-2A and $2 \mathrm{~B}$ ) demonstrated significant disease in LAD (70\% stenosis in the proximal part) and a tight RCA ostial disease. On engaging the RCA and contrast injection, it was found out that a dissection involving the ostial RCA and extending onto the ascending aorta was present. A CT-angiogram(Figure-1) was also available which showed a Type-A Aortic Dissection with the dissection flap involving the RCA ostia and extending till the distal arch of aorta but sparing the arch vessels. Echocardiography revealed massive pericardial effusion. After initial resuscitation, the patient was taken up for surgery. As per our standard protocol of cannulation and conduct of bypass in emergent situations like aortic dissection. We proceeded with distal ascending aortic cannulation using 18 Fr Femoral arterial cannula. Simultaneous exposure of femoral vessels was done but Femoro-Femoral bypass was not used. The ascending aorta was incised and clots were evacuated from the ascending aorta and $\operatorname{arch}($ Figure-3A). Aortic root and aortic valve were normal. The patient underwent an aortic interposition tube graft(30mm Intergard woven Dacron tube graft, Maquet) with CABG(RSVG to distal-RCA and midLAD, Figure-3B). The patient was hemodynamically stable in the post-operative period and was discharged on postoperative day-8. The patient is doing well in the follow-up clinic

\section{DISCUSSION}

IACD complicating coronary interventions is extremely rare and a few cases have been reported. The rapid propagation of aortocoronary dissection may become immediately life-threatening via several sequelae, including hemorrhage into the pericardium resulting in cardiac tamponade, occlusion of the contralateral coronary ostium or propagation of the dissection into the descending aorta. ${ }^{7}$ Most reported iatrogenic aortocoronary dissections have been related to procedures in the RCA, especially during PCI for chronic total occlusions. The RCA is more easily dissected in the retrograde direction into the coronary sinus than the left main coronary artery(LMCA) because of the presence of more smooth muscle cells and a dense matrix of collagen type I fibers ${ }^{8}$ in the peri-ostial wall and sino-tubular junction of the LMCA. Its mechanism involves disruption of the coronary intima by mechanical trauma caused by aggressive manipulation of rigid or hydrophilic guide wires, followed by vigorous contrast injection, which, in turn, contributes to the subsequent retrograde extension of the dissection. Over $40 \%$ of the cases usually spread rapidly to the ascending aorta if the entry-door is not sealed rapidly, a "wait and see" approach may be too risky. To date, the optimal treatment of this rare entity has not been well established. Management depends on the status of the distal coronary circulation and the extent of aortic dissection. When possible dissection can be dealt with deployment of stents distal to the dissection and near the ostium, thus sealing off the entry port. Dunning et al. ${ }^{1}$ categorized aortocoronary dissection according to the level of aortic involvement, where class I denotes dissection involving only the coronaries, class II extending up to $<40 \mathrm{~mm}$ of the ascending aorta, and class III reaching $>40 \mathrm{~mm}$ of the ascending aorta. As class I and II patients with limited involvement of the aorta can benefit from stenting of the coronary dissection entry point without the need for surgical intervention, it was found that urgent surgery is the treatment of choice for class III patients with extensive dissection or patients with hemodynamic instability and those with ischemia of one of the aortic branches.

Aortic dissection when localized may be followed up with ECG and CT scan if coronary blood flow has been corrected by stenting. ${ }^{8}$ However, if the above procedure fails or cannot be attempted without a high risk of further compromising of the coronary circulation as in our case, surgery is the only option. ${ }^{8}$ Surgery was also a preferred option in our case because of the extensive aortic dissection and co-existent coronary artery disease in RCA and LAD which was managed surgically by bypass grafting.

\section{CONCLUSION}

IACD is extremely dangerous and a life-threatening situation requiring emergency surgery; therefore, it is critically important to prevent a catheter-induced aortic dissection during any interventions. Thus, we suggest that surgical management is the preferred modality for this condition. The standard institutional protocols may vary across all institutions but comparable results can be achieved across the globe. Lastly, we suggest that catheter based interventions should be restricted to institutes which are well equipped, especially in view of the current scenario of COVID-19 world pandemic. 


\section{REFERENCES}

1. Dunning DW, Kahn JK, Hawkins ET, O'Neill WW. Iatrogenic coronary artery dissections extending into and involving the aortic root. Catheter Cardiovasc Interv. 2000;51(4):387-393

2. Vega MR. "Aortic dissection: exceedingly rare complication of coronary angioplasty". Cathet Cardiovasc Diagn 1997;42.4:416

3. Ahmed AA, Mahadevan VS, Webb SW, MacGowan SW. "Glue aortoplasty repair of aortic dissection after coronary angioplasty". Annals of Thoracic Surgery 2001;72(3);922-924.

4. Lopez-Minguez JR., et al. "Structural features of sinus of Valsalva and the proximal portion of the coronary arteries: their relevance to retrograde aortocoronary dissection". Revista Española de Cardiología 2006;59(7):696-702.

5. Darwazah AK, Aloul J, Sham'a RAHA, Eida M, Ismail H. "Iatrogenic right coronary artery dissection with retrograde extension to the right coronary sinus". J Card Surg 2008;23(2):177-179

6. Perez-Castellano N, García-Fernández MA, García EJ, Delcán JL. Dissection of the aortic sinus of Valsalva complicating coronary catheterization: cause, mechanism, evolution, and management. Cathet Cardiovasc Diagn. 1998;43(3):273-279

7. Sekiguchi M, Sagawa N, Miyajima A, Hasegawa S, Yamazaki M, Kurabayashi M. Simultaneous right and left coronary occlusion caused by an extensive dissection to the coronary sinus of Valsalva during percutaneous intervention in right coronary artery. Int Heart J. 2009;50(5):663-667.

8. Yip HK, Wu CJ, Yeh KH, Hang CL, Fang CY, Hsieh KY, et al. Unusual complication of retrograde dissection to the coronary sinus of Valsalva during percutaneous revascularization: a single-center experience and literature review. Chest. 2001;119(2):493-501.

\section{FIGURE LEGENDS}

Figure-1-CT scan showing Type-A Aortic Dissection

Figure-2A-Coronary catheterization showing (1)-RCA, (2)-dye spillage suggestive of dissection

Figure-2B-Coronary catheterization showing-(1)LCX, (2)LAD, (3) $\mathrm{OM}_{1}$ with disease in proximal LAD

Figure-3A- Clots in the layers of ascending aorta

3B- Final intra-operative picture showing-(1)RSVG-dRCA, (2)RSVG-mLAD, (3)Interposition Dacron tube graft, (4)Native distal ascending aorta

\section{FIGURES}
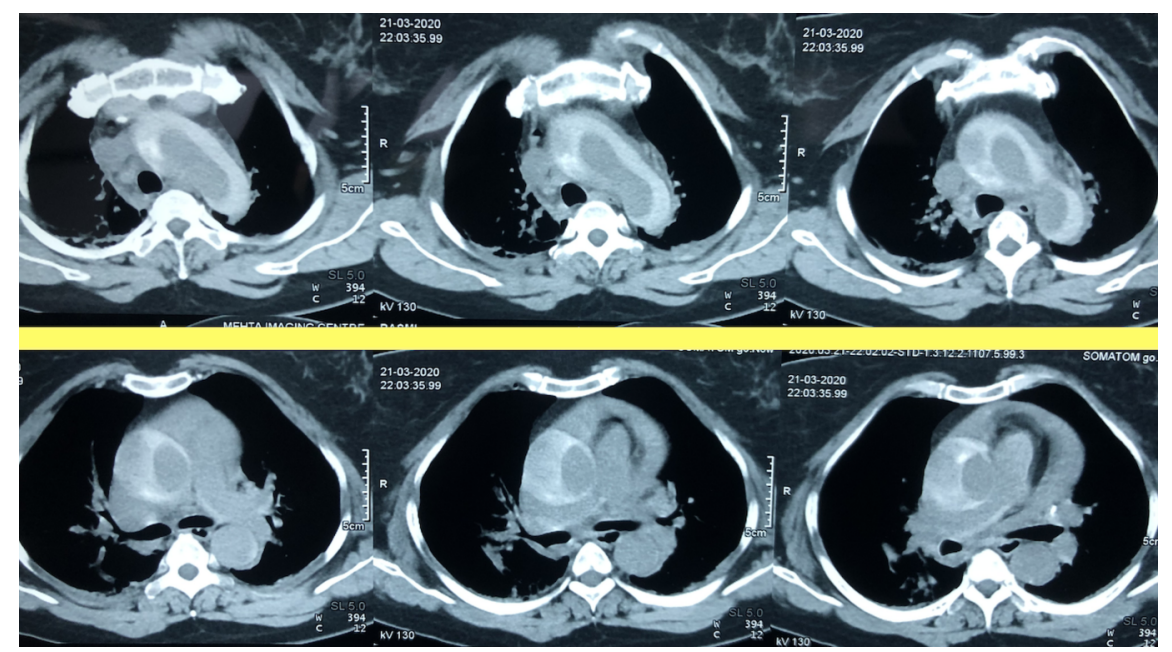

FIGURE-1 


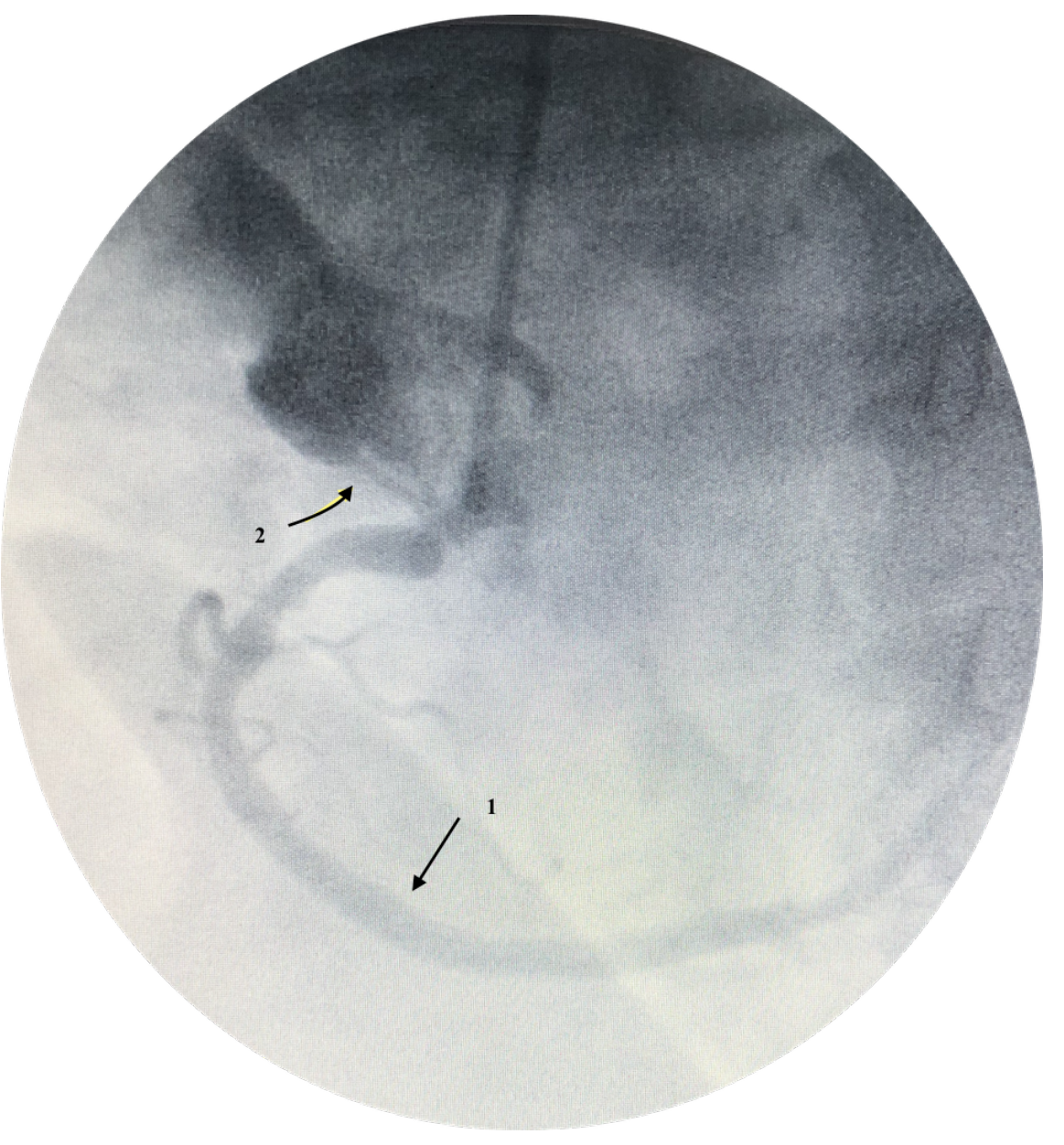

FIGURE-2A 


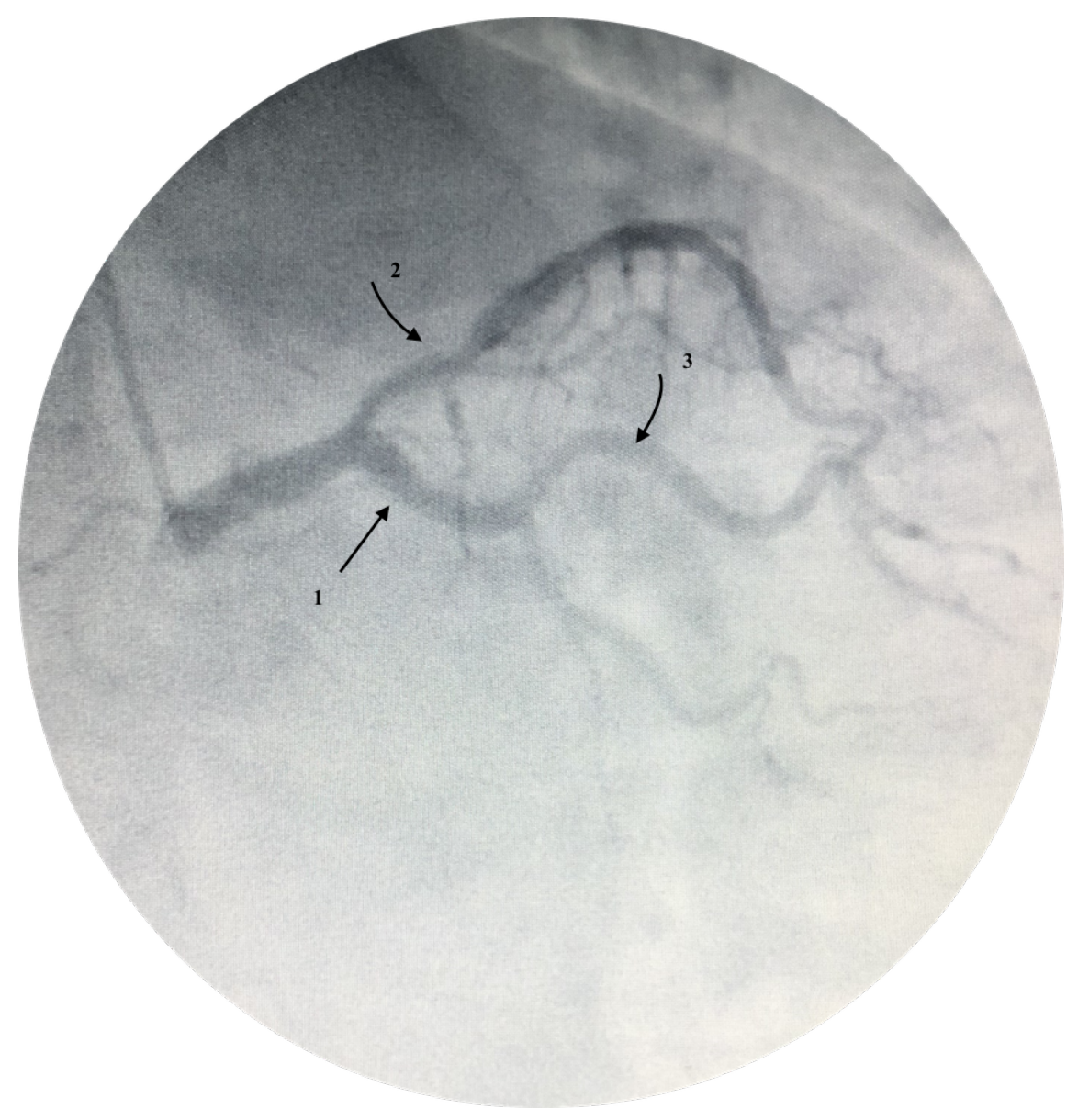

FIGURE-2B

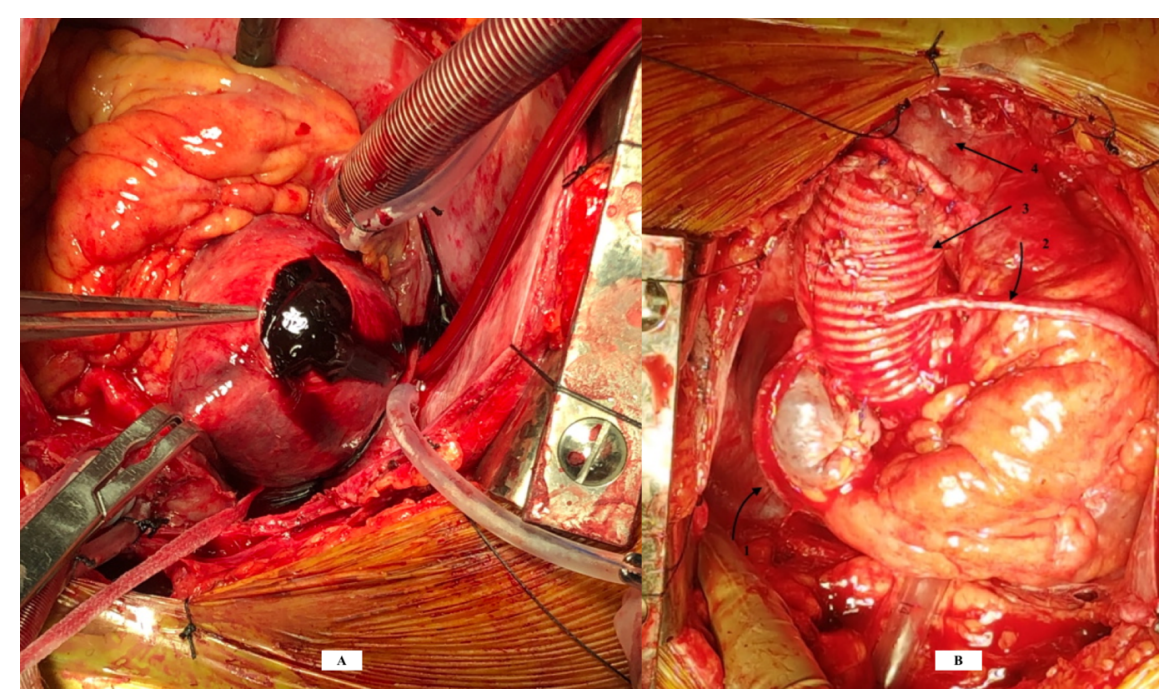

FIGURE-3A \& 3B 